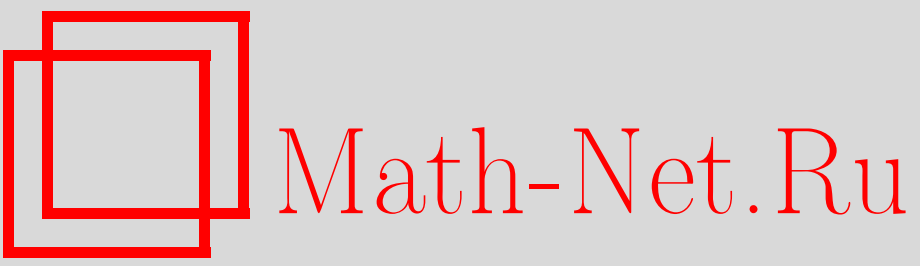

В. М. Ротос, Гомоклинические орбиты возмущенного решеточного модифицированного уравнения КдФ, ТМФ, 2003, том 134, номер 1, 135-147

DOI: https://doi.org/10.4213/tmf146

Использование Общероссийского математического портала Math-Net.Ru подразумевает, что вы прочитали и согласны с пользовательским соглашением

http://www.mathnet.ru/rus/agreement

Параметры загрузки:

IP: 18.208 .226 .222

26 апреля 2023 г., 16:40:20 
ТЕОРЕТИЧЕСКАЯ

И МАТЕМАТИЧЕСКАЯ

ФИЗИКА

Том 134, № 1

январь, 2003

(C) 2003 г.

В. М. Ротос*

\section{ГОМОКЛИНИЧЕСКИЕ ОРБИТЫ ВОЗМУЩЕННОГО РЕШЕТОЧНОГО МОДИФИЦИРОВАННОГО УРАВНЕНИЯ КДФ}

Доказано расщепление гомоклинических орбит для почти интегрируемой решеточной модификации уравнения Кортевега-де Фриза (КдФ) с периодическими граничными условиями. Для построения гомоклинических орбит решеточного модифиицированного уравнения К дФ используется метод преобразований Беклунда. Функция Мельникова строится как градиент инварианта, определенного с помощью дискретного дискриминанта Флоке, вычисленного в критических точках. Установлены критерии сохранения гомоклинических решений при возмущениях решеточной модификации уравнения $\mathrm{K}$ д $\Phi$.

Ключевые слова: гомоклинические решения, решеточное модифицированное уравнение КдФ, метод Мельникова.

\section{1. ВВЕДЕНИЕ}

Нелинейные дисперсионные волновые уравнения дают исследователю замечательные примеры бесконечномерных динамических систем, допускаюших разнообразные и порой удивительные решения типа уединенных волн, образования периодических и сингулярных структур, локализованных структур, периодических по времени, решения, допускаюшие дисперсионную турбулентность, и решения, допускающие пространственно-временное хаотическое поведение [1]. Имеется класс нелинейных волновых уравнений (солитонных уравнений), которые обладают специальным свойством полной интегрируемости и для которых в настояшее время сушествует обширнейшая литература. Примерами таких систем служат уравнение синус-Гордон [2], модифицированное уравнение Кортевега-де Фриза (мКд $\Phi)$ [3] и кубическое нелинейное уравнение Шредингера [4]. При наложенных периодических пространственных граничных условиях эти уравнения допускают частные решения, локализованные в пространстве и гомоклинические по времени.

Чтобы извлечь качественную информацию из моделей, описываемых УрЧП, часто оказывается необходимо применить численные методы решения той или иной задачи, что приводит к необходимости использования разнообразных схем дискретизации. Для

${ }^{*}$ Department of Mathematical Sciences, Loughborough University, Loughborough, UK. E-mail: V.M.Rothos@lboro.ac.uk 
исследования динамики при больших временах качественные характеристики системы приобретают первоочередное значение. В частности, значительный интерес представляет поведение частных решений дискретных моделей в случае, когда интегрируемость нарушается каким-либо (диссипативным или гамильтонова вида) возмушением уравнения. Более точно, при исследовании глобального поведения почти интегрируемых решеточных уравнений мы считаем, что нужно опираться на интуицию, обеспеченную опытом работы в области теории дискретных динамических систем, и применять методы, используемые при исследовании решеточных моделей.

За последние годы достигнут значительньй прогресс в области дискретных интегрируемых систем и связанных с ними разностных уравнений. Значимость дискретных пространственно-временных моделей в математической физике теперь общепризнана. Последуюшее развитие в этой области было в основном обеспечено голландской группой исследователей (см. работу Найхофа и Капеля [5] и приведенную там литературу).

В настоящей работе проводится исследование сушествования гомоклинических орбит для почти интегрируемого решеточного уравнения мКдФ

$$
(p-r) \frac{u_{m+1}^{n}}{u_{m+1}^{n+1}}-(q-r) \frac{u_{m}^{n+1}}{u_{m+1}^{n+1}}=(p+r) \frac{u_{m}^{n+1}}{u_{m}^{n}}-(q+r) \frac{u_{m+1}^{n}}{u_{m}^{n}}
$$

Переменная $u=u_{m}^{n}$ считается динамической (полевой) переменной, определенной в узлах решетки $(n, m) \in \mathbb{Z}$, а числа $p, q \in \mathbb{C}$ считаются параметрами решетки. Уравнение (1.1) получается в подходе непосредственной линеаризации (см. [5] и приведенную там литературу). Сначала рассмотрим интегрируемую решеточную версию уравнения мКдФ, представляющую собой нелинейное УрЧП, т.е. рассмотрим систему, в которой дискретизованы как пространственные, так и временные переменные. Мы покажем, что уравнение с периодическими граничными условиями по дискретному времени допускает решения, которые сходятся гомоклинично по “дискретному" времени к гиперболической фиксированной точке. Решеточное уравнение мКдФ рассматривается как дискретное эволюционное уравнение по отношению к индексу $n$ (в непрерывном пределе этот индекс переходит в переменную времени $t$ ), и будет предполагаться, что полевая переменная $u_{m}^{n}$ удовлетворяет четным периодическим граничным условиям:

$$
\mathcal{L}=\left\{u_{m+M}^{n}=u_{m}^{n}, u_{M-m}^{n}=u_{m}^{n}, M \in 2 \mathbb{Z}_{+}, n \in \mathbb{Z}, m=0,1, \ldots, M-1, h=\frac{2 \pi}{M}\right\} .
$$

В данной работе получены необходимые условия сохранения гомоклинической структуры решений решеточного уравнения мКдФ при малых диссипативных возмушениях. Для этого сначала исследуется вопрос о существовании гомоклинических орбит, связанных с гиперболическими фиксированными точками невозмушенного уравнения с помощью преобразования Беклунда, а затем устанавливается сохранение этих орбит при малых диссипативных возмушениях. Одним из методов, доказавших свою значимость в теории динамических систем, является метод Мельникова [6], [7]. Этот метод задает способ измерения расстояния между стабильными и нестабильными многообразиями, соответствующими некоторому набору инвариантных седловых точек, для возмущенной интегрируемой системы, которая исходно обладала гомоклинической структурой. 
Работа имеет следующую структуру. В разделе 2 содержатся некоторые определения итерационных отображений и приводятся основные положения теории решеточного уравнения мКдФ как интегрируемого нелинейного УрЧП. В разделе 3 получены аналитическое выражение для гомоклинических орбит по дискретному времени $n$ с помощью преобразования Беклунда и формула для градиента отдельной постоянной движения, вычисленной на гомоклинических орбитах. В разделе 4 с помошью метода Мельникова и геометрических соображений выводятся необходимые условия сохранения гомоклинических решений решеточного уравнения мКд $\Phi$ при диссипативных возмущениях.

\section{2. ИНТЕГРИРУЕМОЕ РЕШЕТОЧНОЕ УРАВНЕНИЕ мКДФ}

В данном разделе приводятся некоторые определения из области конечномерных отображений и дается обзор основных свойств решеточного уравнения мКдФ.

2.1. Седловые точки и сепаратрисы. Обозначим через $\mathrm{F}$ некоторый диффеоморфизм. Точка $x_{0}=\mathrm{F}\left(x_{0}\right)$ называется фиксированной точкой отображения $\mathrm{F}$. Фиксированные точки классифицируются в зависимости от спектра матрицы $D F\left(x_{0}\right)$. Особый интерес представляют собой гиперболические фиксированные точки. Фиксированная точка $x_{0}$ называется гиперболической, если хотя бы некоторые из собственных значений матрицы $D \mathrm{~F}\left(x_{0}\right)$ не лежат на единичной окружности. С гиперболическими фиксированными точками можно ассоциировать инвариантные объекты, играющие важную роль в динамике.

Пусть $\delta>0$ - мальй положительный параметр. Множество точек, которые никогда не выйдут за пределы $\delta$-окрестности точки $x_{0}$, называется локальнылм стабильнылм многообразием (или локальной стабильной сепаратрисой):

$$
W_{\mathrm{loc}}^{\mathrm{S}}\left(x_{0}\right)=\left\{x: \forall n \geqslant 0\left\|\mathrm{~F}^{n}-x_{0}\right\| \leqslant \delta\right\} .
$$

Локальным нестабильньцм многообразием (или локальной нестабильной сепаратpисой) называется локальное стабильное многообразие обратного отображения $\mathrm{F}^{-1}$ :

$$
W_{\mathrm{loc}}^{\mathrm{u}}\left(x_{0}\right)=\left\{x: \forall n \geqslant 0\left\|\mathrm{~F}^{-n}-x_{0}\right\| \leqslant \delta\right\} .
$$

Если параметр $\delta$ достаточно мал, то локальное многообразие представляет собой образ единичного круга при некотором гладком (аналитическом) отображении. Размерность круга совпадает с числом собственных значений оператора $D \mathrm{~F}\left(x_{0}\right)$, лежащих вне (для локального нестабильного многообразия) или внутри (для локального стабильного многообразия) единичного круга.

Итерации точек, лежащих на локальной стабильной сепаратрисе, сходятся к фиксированной точке. Это позволяет определить глобальную стабильную сепаратрису как множество всех точек, итерации которых сходятся к фиксированной точке:

$$
W^{\mathrm{S}}\left(x_{0}\right)=\left\{x: \lim _{n \rightarrow+\infty} \mathrm{F}^{n}=x_{0}\right\} .
$$

Будем называть глобальным.м нестабильным многообразием стабильное многообразие обратного отображения $\mathrm{F}^{-1}$ :

$$
W^{\mathrm{u}}\left(x_{0}\right)=\left\{x: \lim _{n \rightarrow-\infty} \mathrm{F}^{n}=x_{0}\right\} .
$$


Глобальные стабильные и нестабильные многообразия могут быть получены итерациями локальных многообразий.

Локальная сепаратриса представляет собой вложение единичного диска. Следовательно, она не имеет самопересечений, поскольку отображение F есть диффеоморфизм, и соответствуюшая глобальная сепаратриса не может пересекаться сама с собой. Легко видеть, что если отображение имеет две гиперболические точки, то их нестабильные (равно как и стабильные) многообразия не пересекаются.

Точка пересечения двух инвариантных многообразий, связанных с одной и той же фиксированной точкой, назьвается гомоклинической точкой. Поскольку оба эти многообразия содержат фиксированную точку $x_{0}$, эта точка исключается из множества гомоклинических точек. Точка называется гетероклинической, если она принадлежит пересечению сепаратрис, связанных с двумя различными фиксированными точками.

2.2. Интегрируемость решеточного уравнения мКдФ. Решеточное уравнение мКд $\Phi(1.1)$ с периодическими граничными условиями следует из дискретной версии принципа наименьшего действия. Действие для решеточного уравнения мКд $\Phi$ имеет вид [8]

$$
\begin{aligned}
S & =\sum_{n \in \mathbb{Z}} \sum_{m=0}^{M-1}\left\{y_{m+1}^{n}\left(y_{m+1}^{n+1}+y_{m}^{n}\right)+\mathrm{F}\left(y_{m}^{n}-y_{m+1}^{n+1}+\sigma_{2}\right)-\mathrm{F}\left(y_{m}^{n}-y_{m+1}^{n+1}+\sigma_{1}\right)\right\} \equiv \\
& \equiv \sum_{n \in \mathbb{Z}} \sum_{m=0}^{M-1} \mathrm{~L}\left(u_{m}^{n}, u_{m+1}^{n}, u_{m+1}^{n+1}, u_{m}^{n+1}\right)
\end{aligned}
$$

где $u_{m}^{n}=e^{y_{m}^{n}}$, функция $\mathrm{F}(x)$ связана с функцией двойного логарифма,

$$
\mathrm{F}(x)=\int_{-\infty}^{x} \ln \left(1+e^{i \xi}\right) d \xi,
$$

а параметры $\sigma_{1}, \sigma_{2}$ связаны с параметрами решетки p, q:

$$
e^{\sigma_{1}}=q \mathrm{p}, \quad e^{\sigma_{2}}=p \mathrm{q} .
$$

Уравнения Эйлера-Лагранжа для действия (2.3) получаются с помошью вариации действия $S$ по переменным $u_{m}^{n}$,

$$
\frac{\delta S}{\delta u_{m}^{n}}=0
$$

Другое важное свойство решеточного уравнения мКд $\Phi$, которое непосредственно связано с интегрируемостью этой системы, - это условие совместности переопределенной вспомогательной линейной системы, известное как пара Лакса [8]:

$$
\begin{aligned}
\phi_{m+1}^{n}(k) & =\mathrm{V}_{n, m}(k) \phi_{m}^{n}, \\
\phi_{m}^{n+1}(k) & =\mathrm{U}_{n, m}(k) \phi_{m}^{n},
\end{aligned}
$$

где $k$ - спектральный параметр, матрица $\mathrm{V}_{n, m}$ имеет вид

$$
\mathrm{V}_{n, m}(k)=\left(\begin{array}{cc}
\frac{\mathrm{q}}{\mathrm{q}-k} & \frac{v_{m+1}^{n}}{\mathrm{q}-k} \\
\frac{k^{2}}{\mathrm{q}-k} \frac{1}{v_{m}^{n}} & \frac{\mathrm{q}}{\mathrm{q}-k} \frac{v_{m+1}^{n}}{v_{m}^{n}}
\end{array}\right),
$$


$\mathrm{a} \mathrm{U}_{n, m}$ - это матрица, получающаяся из $(2.5)$ при заменах $\mathrm{q} \rightarrow \mathrm{p}$ и $(n, m+1) \rightarrow(n+$ $1, m)$.

Обозначим через $\mathrm{M}_{n, m}\left(k, u_{m}^{n}\right)$ фундаментальную матрицу пары Лакса, т.е. матричное решение уравнений (2.4) с начальными условиям $\mathrm{M}_{0}$, представляющими собой единичную матрищу размера $2 \times 2$. Характеристический многочлен

$$
\operatorname{det}\left(\mathrm{M}_{n, m}\left(k, u_{m}^{n}\right)-\lambda \mathrm{I}\right)=0
$$

задает инвариантную алгебраическую кривую. Подстановкой

$$
\mathcal{M}=\lambda-\frac{\operatorname{tr} \mathrm{M}_{n, m}(k)}{2}
$$

можно привести эту кривую к гиперэллиптическому виду:

$$
\mathcal{M}^{2}=\left(\frac{\operatorname{tr} \mathrm{M}_{n, m}(k)}{2}\right)^{2}-\operatorname{det} \mathrm{M}_{n, m}(k) .
$$

Определим функцию $\Delta$ в виде

$$
\Delta: \mathbb{C} \times \mathcal{L} \longrightarrow \mathbb{C}, \quad \Delta\left(k, u_{m}^{n}\right)=\operatorname{tr} \mathrm{M}_{n, m}\left(M ; k, u_{m}^{n}\right) .
$$

Спектральный параметр $k \in \mathbb{C}$ удовлетворяет условию

$$
-2 \mathrm{D} \leqslant \Delta\left(k, u_{m}^{n}\right) \leqslant 2 \mathrm{D},
$$

где $\mathrm{D}$ - константа, а критическая точка $k_{\mathrm{c}}$ функции $\Delta$ задается условием

$$
\left.\frac{d \Delta\left(k, u_{m}^{n}\right)}{d k}\right|_{k=k^{\mathrm{c}}}=0 .
$$

Важное следствие, вытекающее из интеграла движения $\mathcal{F}_{j}: \mathcal{L} \rightarrow \mathbb{C}$, имеет вид

$$
\mathcal{F}_{j}\left(u_{m}^{n}\right)=\frac{1}{\mathrm{D}} \Delta\left(k_{j}^{\mathrm{c}}, u_{m}^{n}\right) .
$$

\section{3. АНАЛИЗ СТАБИЛЬНОСТИ И ГОМОКЛИНИЧЕСКИЕ ОРБИТЫ}

3.1. Анализ линеаризованного уравнения. Ясно, что фиксированные решения имеют вид $\hat{u}_{m}^{n}=e^{2(-1)^{m}} i \pi$. Предположим, что малые возмушения $\hat{u}_{m}^{n}(\varepsilon)$ растут экспоненциально быстро ввиду присутствия на пространственном отрезке $(0,1)$ линейно нестабильных мод непрерывного уравнения мКд $\Phi$ :

$$
\begin{aligned}
& u_{m}^{n}=\hat{u}_{m}^{n}+\eta \varphi_{m}^{n}, \quad 0<\eta \ll 1, \\
& \varphi_{m}^{n}=\Omega_{k}^{n} e^{i \mathcal{A}_{k} m}, \quad \mathcal{A}_{k}=\frac{2 \pi k}{M},
\end{aligned}
$$

и $\hat{u}_{m}^{n}$ есть решение уравнения (1.1), $M \in 2 \mathbb{Z}_{+}-$полное число узлов решетки в пространственном интервале $(0,1)$ и $k=-M / 2, \ldots,(M / 2)-1$.

Линеаризуя уравнение (1.1), получим

$$
p\left(\varphi_{m+1}^{n}+\varphi_{m}^{n}\right)+q\left(\varphi_{m+1}^{n}+\varphi_{m+1}^{n+1}\right)=p\left(\varphi_{m+1}^{n+1}+\varphi_{m}^{n+1}\right)+q\left(\varphi_{m}^{n+1}+\varphi_{m}^{n}\right) .
$$


С помощью прямых вычислений можно получить уравнение на собственные значения $\Omega_{k}$ оператора отображения:

$$
\Omega_{k}=\frac{\mathcal{B}+\cos \mathcal{A}_{k}}{1+\mathcal{B} \cos \mathcal{A}_{k}}+i \frac{-p q\left(p^{2}+q^{2}\right)^{-1} \sin \mathcal{A}_{k}}{1+\mathcal{B} \cos \mathcal{A}_{k}}
$$

где

$$
\mathcal{B}=\frac{p^{2}-q^{2}}{p^{2}+q^{2}}, \quad h=\frac{2 \pi}{M} \ll 1 .
$$

Условие стабильности $\left|\Omega_{k}\right| \leqslant 1$ при этом принимает вид $\cos \mathcal{A}_{k} \leqslant 1$. Оно нарушается для волновых чисел $\mathcal{A}_{k} \simeq 0$ при $k=0$. Нестабильными волновыми числами называются те числа, при которых $\left|\Omega_{k}\right| \geqslant 1$.

3.2. Аналитические формулы для гомоклинических орбит. Будем строить гомоклинические орбиты для гиперболических фиксированных точек решеточного уравнения мКдФ с помошью преобразования Беклунда (детальное описание преобразования Беклунда можно найти, например, в книге [9]). Невозмушенное решеточное уравнение мКдФ имеет нестабильную гиперболическую фиксированную точку $\hat{u}_{m}^{n}$. В конкретном примере преобразования Беклунда будут использованы для построения гомоклинических орбит с помошью итераций преобразования, в которых будут принимать участие все моды - нестабильные и стабильные.

Обозначим через $\phi_{m}^{n}$ фиксированное решение пары Лакса в точке $\left(k_{\mathrm{d}}, u_{m}^{n}\right)$, где $k_{\mathrm{d}}-$ двойная критическая точка функции $\Delta$, т.е. это решение удовлетворяет условию (2.10), и при этом $\Delta\left(k_{\mathrm{d}}, u_{m}^{n}\right)= \pm 2 \mathrm{D}$. Зададим $(2 \times 2)$-матрицу преобразования $\Gamma_{n, m}$ в виде

$$
\Gamma_{n, m}=\left(\begin{array}{cc}
-\bar{k}-\alpha_{n, m} & \beta_{n, m} \\
\gamma_{n, m} & -\bar{k}-\delta_{n, m}
\end{array}\right)
$$

где

$$
\begin{gathered}
\alpha_{n, m}=\frac{k}{\mathrm{D}}\left(\left|\phi_{m}^{n, 1}\right|^{2}+\bar{k}^{2}\left|\phi_{m}^{n, 2}\right|^{2}\right), \quad \delta_{n, m}=\bar{\alpha}_{n, m}, \\
\beta_{n, m}=\frac{\phi_{m}^{n, 1} \bar{\phi}_{m}^{n, 2}}{\mathrm{D}}\left(\bar{k}^{2}-|k|^{2}\right), \quad \gamma_{n, m}=-\bar{\beta}_{n, m}, \quad \mathrm{D}=\left|\phi_{m}^{n, 1}\right|^{2}+|k|^{2}\left|\phi_{m}^{n, 2}\right|^{2} .
\end{gathered}
$$

Также введем определения

$$
\begin{gathered}
\mathrm{U}_{m}^{n}=\mathrm{p}\left(\bar{k}+\alpha_{n-1, m}+\left(1-\frac{k^{2}}{\mathrm{p}^{2}}\right)+\frac{\mathrm{p}-k}{v_{m}^{n}} \beta_{n, m}\right) \times \\
\times\left(-\bar{\beta}_{n-1, m}+\frac{\mathrm{p}-k}{\mathrm{p}} \frac{\bar{k}+\bar{\alpha}_{n-1, m}}{v_{m}^{n-1}}\right)^{-1}, \\
\Phi_{m}^{n}(k)=\Gamma_{n, m}\left(k_{\mathrm{d}}\right) \phi_{m}^{n}(k) .
\end{gathered}
$$


ПРЕДЛОЖЕНИЕ 1. Пусть $u_{m}^{n}$ - решение решеточного уравнения мКдФ с двойной критической точкой $k_{\mathrm{d}}$, а $\phi_{m}^{n}$ - общее решение пары Лакса в точке $\left(k_{\mathrm{d}}, u_{m}^{n}\right)$. Определим функиии $\mathrm{U}_{m}^{n} u \Phi_{m}^{n}(k)$ с помощью формул (3.7) и (3.8). Тогда получим, что:

1) функиия $\mathrm{U}_{m}^{n}$ также представляет собой решение решеточного уравнения мKठФ ;

2) $\Phi_{m}^{n}(k)$ дает решение пары Лакса $(2.4)$ в точке $\left(k, \mathrm{U}_{m}^{n}\right)$;

3) последовательность $\mathrm{U}_{m}^{n}$ гомоклинично сходится $к$ фиксированному решению $\hat{u}_{m}^{n}, \operatorname{ma\kappa }$ чmo $\lim _{n \rightarrow \pm \infty} \mathrm{U}_{m}^{n}=e^{2(-1)^{m} i \pi}$.

ДокАЗАТЕЛЬСтво. Покажем, что функция $\Phi_{m}^{n}(k)$ задает решение пары Лакса в точке $\left(k, \mathrm{U}_{m}^{n}\right)$ при условии, что функция $\phi_{m}^{n}$ задает решение уравнений $(2.4)$ в точке $\left(k, u_{m}^{n}\right)$. Из уравнений пары Лакса получим

$$
\begin{aligned}
\Phi_{m+1}^{n}-\mathrm{V}_{n, m}\left(\mathrm{U}_{m}^{n}\right) \Phi_{m}^{n} & =\left[\Gamma_{n, m+1} \mathrm{~V}_{n, m}\left(u_{m}^{n}\right)-\mathrm{V}_{n, m}\left(\mathrm{U}_{m}^{n}\right) \Gamma_{n, m}\right] \Phi_{m}^{n} \\
\Phi_{m}^{n+1}-\mathrm{U}_{n, m}\left(\mathrm{U}_{m}^{n}\right) \Phi_{m}^{n} & =\left[\Gamma_{n+1, m} \mathrm{U}_{n, m}\left(u_{m}^{n}\right)-\mathrm{U}_{n, m}\left(\mathrm{U}_{m}^{n}\right) \Gamma_{n, m}\right] \Phi_{m}^{n}
\end{aligned}
$$

Таким образом, если выполняются равенства

$$
\begin{aligned}
& \Gamma_{n, m+1} \mathrm{~V}_{n, m}\left(u_{m}^{n}\right)-\mathrm{V}_{n, m}\left(\mathrm{U}_{m}^{n}\right) \Gamma_{n, m}=0, \\
& \Gamma_{n+1, m} \mathrm{U}_{n, m}\left(u_{m}^{n}\right)-\mathrm{U}_{n, m}\left(\mathrm{U}_{m}^{n}\right) \Gamma_{n, m}=0,
\end{aligned}
$$

то предложение будет доказано. В компонентной форме записи уравнения (3.9) вместе с определениями (3.5), (3.7) оказьваются эквивалентными системе из восьми алгебраических уравнений. В самом деле, система

$$
\begin{aligned}
& \Gamma_{n, m+1}=\mathrm{V}_{n, m}\left(\mathrm{U}_{m}^{n}\right) \Gamma_{n, m} \mathrm{~V}_{n, m}^{-1}\left(u_{m}^{n}\right), \\
& \Gamma_{n+1, m}=\mathrm{U}_{n, m}\left(\mathrm{U}_{m}^{n}\right) \Gamma_{n, m} \mathrm{U}_{n, m}^{-1}\left(u_{m}^{n}\right)
\end{aligned}
$$

задает следуюшую систему алгебраических уравнений:

$$
\begin{aligned}
\mathcal{P}\left(\bar{k}+\alpha_{n+1, m}\right)= & \left(\bar{k}+\alpha_{n, m}+\frac{\mathrm{p}-k}{\mathrm{p}} \frac{1}{v_{m}^{n+1}} \beta_{n, m}\right)- \\
& -\frac{\mathrm{U}_{m}^{n+1}}{\mathrm{p}}\left(\gamma_{n, m}+(\mathrm{p}-k) \frac{\bar{k}+\delta_{n, m}}{\mathrm{p} v_{m}^{n+1}}\right), \\
\mathcal{P} \beta_{n+1, m}= & \left(\bar{k}+\alpha_{n, m}\right) \frac{v_{m}^{n}}{\mathrm{p}}+\beta_{n, m} \frac{v_{m}^{n}}{v_{m}^{n+1}}- \\
& -\frac{\mathrm{U}_{m}^{n+1}}{\mathrm{p}}\left(\bar{\beta}_{n, m} \frac{v_{m}^{n}}{\mathrm{p}}+\left(\bar{k}+\bar{\alpha}_{n, m}\right) \frac{v_{m}^{n}}{v_{m}^{n+1}}\right), \\
\mathcal{P} \gamma_{n+1, m}= & -\frac{k^{2}}{\mathrm{p}} \frac{1}{\mathrm{U}_{m}^{n}}\left(\bar{k}+\alpha_{n, m}+\frac{\beta_{n, m}}{v_{m}^{n+1}} \frac{\mathrm{p}}{\mathrm{p}-k}\right)+ \\
& +\frac{\mathrm{U}_{m}^{n+1}}{\mathrm{U}_{m}^{n}}\left(\gamma_{n, m}+\frac{\bar{k}+\delta_{n, m}}{v^{n, m}} \frac{\mathrm{p}-k}{\mathrm{p}}\right), \\
\mathcal{P}\left(\bar{k}+\delta_{n+1, m}\right)= & -\frac{k^{2}}{\mathrm{p} \mathrm{U}_{m}^{n}}\left(\left(\bar{k}+\alpha_{n, m}\right) \frac{v_{m}^{n}}{\mathrm{p}}+\beta_{n, m} \frac{v_{m}^{n}}{v_{m}^{n+1}}\right)+
\end{aligned}
$$




$$
\begin{aligned}
& +\frac{\mathrm{U}_{m}^{n+1}}{\mathrm{U}_{m}^{n}}\left(\gamma_{n, m} \frac{v_{m}^{n}}{\mathrm{p}}+\left(\bar{k}+\delta_{n, m}\right) \frac{v_{m}^{n}}{v_{m}^{n+1}}\right), \\
\mathcal{Q}\left(\bar{k}+\alpha_{n, m+1}\right)= & \left(\bar{k}+\alpha_{n, m}+\frac{\mathrm{q}-k}{\mathrm{q}} \frac{\beta_{n, m+1}}{v_{m}^{n+1}}\right)- \\
& -\frac{\mathrm{U}_{m+1}^{n}}{\mathrm{q}}\left(\gamma_{n, m}+\frac{\mathrm{q}-k}{\mathrm{q}} \frac{\bar{k}+\delta_{n, m}}{v_{m+1}^{n}}\right), \\
\mathcal{Q} \beta_{n, m+1}= & \left.\left(\bar{k}+\alpha_{n, m}\right) \frac{v_{m}^{n}}{\mathrm{q}}+\beta_{n, m} \frac{v_{m}^{n}}{v_{m+1}^{n}}\right)- \\
& -\frac{\mathrm{U}_{m+1}^{n}}{\mathrm{q}}\left(\gamma_{n, m} \frac{v_{m}^{n}}{\mathrm{q}}+\left(\bar{k}+\delta_{n, m}\right) \frac{v_{m}^{n}}{v_{m+1}^{n}}\right), \\
\mathcal{Q} \gamma_{n, m+1}=- & \frac{k^{2}}{\mathrm{q} \mathrm{U}_{m}^{n}}\left(\bar{k}+\alpha_{n, m}+\frac{\mathrm{q}-k}{\mathrm{q}} \frac{\beta_{n, m}}{v_{m+1}^{n}}\right)+ \\
& +\frac{\mathrm{U}_{m+1}^{n}}{\mathrm{U}_{m}^{n}}\left(\gamma_{n, m}+\frac{\mathrm{q}-k}{\mathrm{q}} \frac{\bar{k}+\delta_{n, m}}{v_{m+1}^{n}}\right), \\
\mathcal{Q}\left(\bar{k}+\delta_{n, m+1}\right)= & \frac{k^{2}}{\mathrm{q} \mathrm{U}_{m}^{n}}\left(\left(\bar{k}+\alpha_{n, m}\right) \frac{v_{m}^{n}}{\mathrm{q}}+\frac{v_{m}^{n}}{v_{m+1}^{n}} \beta_{n, m}\right)+ \\
& +\frac{\mathrm{U}_{m}^{n+1}}{\mathrm{U}_{m}^{n}}\left(\gamma_{n, m} \frac{v_{m}^{n}}{\mathrm{q}}+\left(\bar{k}+\delta_{n, m}\right) \frac{v_{m}^{n}}{v_{m+1}^{n}}\right),
\end{aligned}
$$

где

$$
\mathcal{P}=\left(1-\frac{k^{2}}{\mathrm{p}^{2}}\right)^{-1}, \quad \mathcal{Q}=\left(1-\frac{k^{2}}{\mathrm{q}^{2}}\right)^{-1} .
$$

Таким образом, задача свелась к проверке справедливости этих восьми уравнений. Это непосредственно следует из представлений для величин $\alpha_{n, m}, \beta_{n, m}, \gamma_{n, m}, \delta_{n, m} \mathbf{U}_{m}^{n}$.

Рассмотрим теперь $u_{m}^{n}=\hat{u}_{m}^{n}$ (фиксированную точку) как потенциальную функцию в паре Лакса; тогда решения уравнений (2.4) примут вид

$$
\phi_{m}^{n(-)}=\left(\begin{array}{c}
1 \\
-k
\end{array}\right) \lambda_{-}^{m}, \quad \phi_{m}^{n(+)}=\left(\begin{array}{c}
1 \\
k
\end{array}\right) b^{n} \lambda_{+}^{m},
$$

где

$$
\lambda_{ \pm}=a \pm \frac{k a}{\mathrm{q}}, \quad a:=\frac{\mathrm{q}}{\mathrm{q}-k}, \quad b:=\frac{\mathrm{p}+k}{\mathrm{p}-k},
$$

и $k=k_{\mathrm{d}}$ есть двойная критическая точка отображения $\Delta$, т.е. получим, что

$$
\Delta(k)=2 \mathrm{D}, \quad \frac{d}{d k} \Delta(k)=0,
$$

где

$$
\Delta(k)=\left(a-\frac{k a}{\mathrm{q}}\right)^{m}+b^{n} k\left(a+\frac{k a}{\mathrm{q}}\right)^{m}, \quad\left|\mathrm{M}_{n, m}\right|:=2 b^{n} k\left(a^{2}-\left(\frac{k a}{\mathrm{q}}\right)^{2}\right)^{m}
$$

$$
\operatorname{det} \mathrm{M}_{n, M}\left(\phi^{-}, \phi^{+}\right)=\mathrm{D}^{2} \operatorname{det} \mathrm{M}_{n, 0}, \quad \mathrm{D}^{2}:=\prod_{m=0}^{M-1}\left(\lambda_{-} \lambda_{+}\right)^{m}
$$


Подставляя выражения (3.11) в формулу (3.7), получим следуюшие аналитические выражения для гомоклинических орбит:

$$
\begin{aligned}
\mathrm{U}_{m}^{n}= & e^{2(-1)^{m} i \pi} \times \\
& \times \frac{1-\mathrm{P}^{2} \cos ^{2}\left(m h \mathrm{w}_{1}+\mathrm{x}\right) \operatorname{sech}^{2}\left(n h \mathrm{w}_{2}+\tau\right)+2 i \mathrm{P} \cos \left(m h \mathrm{w}_{1}+\mathrm{x}\right) \operatorname{sech}\left(n h \mathrm{w}_{2}+\tau\right)}{1+\mathrm{P}^{2} \cos ^{2}\left(m h \mathrm{w}_{1}+\mathrm{x}\right) \operatorname{sech}^{2}\left(n h \mathrm{w}_{2}+\tau\right)},
\end{aligned}
$$

где

$$
\mathrm{P}=\frac{\operatorname{sh} \mathrm{w}_{2} h}{\sin \mathrm{w}_{1} h}, \quad h \ll 1,
$$

$\mathrm{w}_{1,2}$ - некоторые постоянные, $\lim _{n \rightarrow \pm \infty} \mathrm{U}_{m}^{n}=\hat{u}_{m}^{n}$.

3.3. Градиент инварианта $\Delta\left(k, u_{m}^{n}\right)$. Здесь мы выведем явную формулу для градиента важного интеграла движения, которьй в дальнейшем используется для измерения функции расстояния между инвариантными многообразиями.

След фундаментальной матрицы пары Лакса представляет собой важный интеграл движения для уравнения синус-Гордон. Соответствуюшие инварианты $\mathcal{F}_{j}\left(u_{m}^{n}\right)$ для решеточного уравнения мКд $\Phi$ имеют вид

$$
\mathcal{F}_{j}\left(u_{m}^{n}\right)=\frac{1}{\mathrm{D}} \Delta\left(k_{j}^{\mathrm{c}}\left(u_{m}^{n}\right), u_{m}^{n}, u_{m+1}^{n}\right) .
$$

Инварианты $\mathcal{F}_{j}$ используются в разделе 4 для построения функций Мельникова.

ЛЕммА. Пусть $k_{j}^{\mathrm{c}}\left(u_{m}^{n}, u_{m+1}^{n}\right)$ - простая критическая точка преобразования $\Delta$. Тогда

$$
\begin{aligned}
\frac{\delta \mathcal{F}_{j}\left(u_{m}^{n}, u_{m+1}^{n}\right)}{\delta u_{m}^{n}} & =\frac{1}{\mathrm{D}} \frac{\delta \Delta\left(k_{j}^{\mathrm{c}}\left(u_{m}^{n}\right), u_{m}^{n}, u_{m+1}^{n}\right)}{\delta u_{m}^{n}} \\
\frac{\delta \mathcal{F}_{j}\left(u_{m}^{n}, u_{m+1}^{n}\right)}{\delta u_{m+1}^{n}} & =\frac{1}{\mathrm{D}} \frac{\delta \Delta\left(k_{j}^{\mathrm{c}}\left(u_{m}^{n}\right), u_{m}^{n}, u_{m+1}^{n}\right)}{\delta u_{m+1}^{n}}
\end{aligned}
$$

$2 \partial e$

$$
\begin{aligned}
& \frac{\delta \Delta\left(k, u_{m}^{n}\right)}{\delta u_{m}^{n}}=\frac{2 i}{\mathrm{q}-k} \operatorname{tr}\left[\mathrm{M}_{n, m+1}^{-1}\left(\begin{array}{cc}
0 & 0 \\
\frac{k^{2}}{v_{m}^{n}} & -\mathrm{q} \frac{v_{m+1}^{n}}{v_{m}^{n}}
\end{array}\right) \mathrm{M}_{n, m} \mathrm{M}_{n, M}\right], \\
& \frac{\delta \Delta\left(k, u_{m}^{n}\right)}{\delta u_{m+1}^{n}}=\frac{2 i}{\mathrm{q}-k} \operatorname{tr}\left[\mathrm{M}_{n, m+1}^{-1}\left(\begin{array}{cc}
0 & v_{m+1}^{n} \\
0 & \mathrm{q} \frac{v_{m+1}^{n}}{v_{m}^{n}}
\end{array}\right) \mathrm{M}_{n, m} \mathrm{M}_{n, M}\right],
\end{aligned}
$$

$v_{m}^{n}=e^{2 i u_{m}^{n}} u \mathrm{M}_{n, m}=\left[\phi_{m}^{n(-)}, \phi_{m}^{n(+)}\right]$. Здесь $\phi_{m}^{n( \pm)}=\left(\phi_{m}^{n( \pm 1)}, \phi_{m}^{n( \pm 2)}\right)$ суть две функчии Блоха для пары Лакса (2.4) и $\left|\mathrm{M}_{n, m}\right|=\operatorname{det} \mathrm{M}_{n, m}$.

ДокАЗАТЕЛЬСтво. Поскольку $k_{j}^{\mathrm{c}}$ есть критическая точка, то $\Delta^{\prime}\left(k_{j}^{\mathrm{c}}\left(u_{m}^{n}\right), u_{m}^{n}\right)=0$ и

$$
\frac{\delta k_{j}^{c}}{\delta u_{m}^{n}}=-\frac{1}{\Delta^{\prime \prime}} \frac{\delta \Delta^{\prime}}{\delta u_{m}^{n}} .
$$

Тогда $k_{j}^{c}-$ дифференциальная функция,

$$
\mathrm{D} \frac{\delta \mathcal{F}_{j}\left(k, u_{m}^{n}\right)}{\delta u_{m}^{n}}=\left.\frac{\delta \Delta}{\delta u_{m}^{n}}\right|_{k=k_{j}^{\mathrm{c}}}+\left.\frac{\delta \Delta}{\delta k}\right|_{k=k_{j}^{\mathrm{c}}} \frac{\delta k_{j}^{\mathrm{c}}}{\delta u_{m}^{n}}=\left.\frac{\delta \Delta}{\delta u_{m}^{n}}\right|_{k=k_{j}^{\mathrm{c}}} .
$$


Обозначим через $\mathrm{M}_{n, m}$ фундаментальную матрицу пары Лакса с потенциальной функцией $v^{n}$. Варьируя $v_{m}^{n}$, можно получить вариационное уравнение на вариацию $\mathrm{M}_{n, m}$ при фиксированном $k$ :

$$
\begin{aligned}
\mathrm{M}_{n, m+1} & =\mathrm{V}_{n, m} \mathrm{M}_{n, m}, \\
\delta \mathrm{M}_{n, m+1} & =\mathrm{V}_{n, m} \delta \mathrm{M}_{n, m}+\delta \mathrm{V}_{n, m} \mathrm{M}_{n, m}, \\
\delta \mathrm{V}_{n, m} & =\left(\begin{array}{cc}
0 & \frac{1}{\mathrm{q}-k} \delta v_{m+1}^{n} \\
\frac{k^{2}}{\mathrm{q}-k} \delta\left(\frac{1}{v_{m}^{n}}\right) & \frac{\mathrm{q}}{\mathrm{q}-k} \delta\left(\frac{v_{m+1}^{n}}{v_{m}^{n}}\right)
\end{array}\right) .
\end{aligned}
$$

Пусть $\delta \mathrm{M}_{n, m}=\mathrm{M}_{n, m} \mathrm{~A}_{n, m}$, где $\mathrm{A}_{n, m}$ - неизвестная матрица размера $2 \times 2$, которую надо найти,

$$
\begin{aligned}
\delta \mathrm{M}_{n, m+1} & =\mathrm{M}_{n, m+1} \mathrm{~A}_{n, m+1}=\mathrm{V}_{n, m} \mathrm{M}_{n, m} \mathrm{~A}_{n, m}+\delta \mathrm{V}_{n, m} \mathrm{M}_{n, m}= \\
& =\mathrm{M}_{n, m+1} \mathrm{~A}_{n, m}+\delta \mathrm{V}_{n, m} \mathrm{M}_{n, m} .
\end{aligned}
$$

Тем самым получим

$$
\mathrm{A}_{n, m+1}-\mathrm{A}_{n, m}=\mathrm{M}_{n, m+1}^{-1} \delta \mathrm{V}_{n, m} \mathrm{M}_{n, m}, \quad \mathrm{~A}_{n, 0}=0 .
$$

Решая систему (3.18), получим формулу

$$
\delta \mathrm{M}_{n, m}=\mathrm{M}_{n, M}\left[\sum_{j=1}^{M-1} \mathrm{M}_{n, j}^{-1} \delta \mathrm{V}_{n, j-1} \mathrm{M}_{n, j-1}\right], \quad \delta \mathrm{M}_{n, 0}=0 .
$$

Тогда

$$
\delta \Delta\left(k, u_{m}^{n}\right)=\operatorname{tr}\left\{\mathrm{M}_{n, M}\left[\sum_{j=1}^{M-1} \mathrm{M}_{n, j}^{-1} \delta \mathrm{V}_{n, j-1} \mathrm{M}_{n, j-1}\right]\right\},
$$

и в итоге получаются уравнения (3.17). Подставляя представление для $\mathrm{M}_{n, m}$ в формулы (3.17), получим вариационные выражения

$$
\begin{aligned}
\frac{\delta \Delta\left(k, u_{m}^{n}\right)}{\delta u_{m}^{n}}= & \frac{2 i}{\mathrm{q}-k} \frac{1}{\left|\mathrm{M}_{n, m+1}\right|}\left\{-\frac{k^{2}}{v_{m}^{n}}\left[\phi_{m+1}^{n(+1)}\left(\phi_{m}^{n(-1)} \phi_{M}^{n(-1)}+\phi_{m}^{n(+1)} \phi_{M}^{n(-2)}\right)+\right.\right. \\
& \left.+\phi_{m+1}^{n(-2)}\left(\phi_{m}^{n(-1)} \phi_{M}^{n(+1)}+\phi_{m}^{n(+1)} \phi_{M}^{n(+2)}\right)\right]+ \\
& +\mathrm{q} \frac{v_{m+1}^{n}}{v_{m}^{n}}\left[\phi _ { m + 1 } ^ { n ( - 1 ) } \left(\phi_{m}^{n(-1)} \phi_{M}^{n(+1)}+\right.\right. \\
& \left.\left.\left.+\phi_{m}^{n(+2)} \phi_{M}^{n(+2)}\right)-\phi_{m+1}^{n(+1)}\left(\phi_{m}^{n(-2)} \phi_{M}^{n(-1)}+\phi_{m}^{n(+2)} \phi_{M}^{n(-2)}\right)\right]\right\} \\
\frac{\delta \Delta\left(k, u_{m}^{n}\right)}{\delta u_{m+1}^{n}=} & \frac{2 i}{\mathrm{q}-k} \frac{1}{\left|\mathrm{M}_{n, m+1}\right|}\left\{v _ { m + 1 } ^ { n } \left[\phi_{m}^{n(+2)}\left(\phi_{m}^{n(-2)} \phi_{M}^{n(-1)}+\phi_{m}^{n(+2)} \phi_{M}^{n(-2)}\right)-\right.\right. \\
& \left.-\phi_{m}^{n(-2)}\left(\phi_{m}^{n(-2)} \phi_{M}^{n(+1)}+\phi_{m}^{n(+2)} \phi_{M}^{n(+2)}\right)\right]+ \\
& +\mathrm{q} \frac{v_{m+1}^{n}}{v_{m}^{n}}\left[\phi _ { m } ^ { n ( - 1 ) } \left(\phi_{m}^{n(-2)} \phi_{M}^{n(+1)}+\right.\right. \\
& \left.\left.\left.+\phi_{m}^{n(+2)} \phi_{M}^{n(+2)}\right)-\phi_{m}^{n(+1)}\left(\phi_{m}^{n(-2)} \phi_{M}^{n(-1)}+\phi_{m}^{n(+2)} \phi_{M}^{n(-2)}\right)\right]\right\}
\end{aligned}
$$

Подставляя теперь выражения (3.11) и (3.14) в формулы (3.19), получим формулы для вариации $\delta \Delta / \delta u_{m}^{n}$, вычисленной на гомоклинических орбитах. Это завершает доказательство леммы. 


\section{4. ГОМОКЛИНИЧЕСКИЕ ОРБИТЫ ДЛЯ ВОЗМУШЕННОГО РЕШЕТОЧНОГО УРАВНЕНИЯ МКДФ}

Установим теперь сохранение свойства гомоклиничности для орбит (3.14) возмушенного решеточного уравнения мКд $\Phi$

$$
u_{m}^{n+1}=\mathbf{F}\left(u_{m}^{n-1}, u_{m \pm 1}^{n}\right)+\varepsilon \mathbf{G}\left(u_{m}^{n}, u_{m \pm 1}^{n}\right),
$$

где $\mathbf{G}$ обозначает возмушение,

$$
\mathbf{G}:=\operatorname{bg}_{1}\left(u_{m}^{n}\right)+\operatorname{cg}_{2}\left(u_{m \pm 1}^{n \pm 1}\right) .
$$

Используя ранее построенные геометрические структуры, будем строить орбиты возмушенного решеточного уравнения мКд $\Phi$ (4.1), которые обладают свойством гомоклиничности по отношению к гиперболической (фиксированной) точке $\hat{u}_{m}^{n}(\varepsilon)$. Это построение осуществляется в два этапа. Пусть задана начальная точка в нестабильном многообразии точки $\hat{u}_{m}^{n}(\varepsilon)$. Покажем, что орбиты в положительном направлении попадают в центрально-стабильное многообразие $W_{\varepsilon}^{\mathrm{cs}}$ окрестности $\mathcal{M}_{\varepsilon}$ при подходяшим образом выбранных параметрах b, с. Затем покажем, что возмушенные орбиты сходятся к фиксированной точке $\hat{u}_{m}^{n}(\varepsilon)$ в положительном направлении по времени $n \rightarrow+\infty$. При этом основным используемым при доказательстве методом является метод Мельникова.

Возмушенная система имеет фиксированную седловую точку $\hat{u}_{m}^{n}(\varepsilon)$, в то время как анализ линейной стабильности в полном фазовом пространстве показывает, что точка $\hat{u}_{m}^{n}(\varepsilon)$ действительно седловая, а из теории инвариантных многообразий следует, что область $W^{\mathrm{u}}\left(\hat{u}_{m}^{n}(\varepsilon)\right)$ сушествует и имеет размерность 2 . Область $W^{\mathrm{s}}\left(\hat{u}_{m}^{n}(\varepsilon)\right)$ при этом также сушествует и имеет коразмерность 2 , а область $W_{\varepsilon}^{\text {cs }}$ имеет коразмерность 1 . Область перекрытия $W^{\mathrm{u}}\left(\hat{u}_{m}^{n}(\varepsilon)\right) \cap W_{\varepsilon}^{\mathrm{cs}}$ при этом оказывается одномерной.

Таким образом, нестабильные, стабильные и центрально-стабильные многообразия отображения $\mathbf{F}$ (ср. с решеточным уравнением мKд $\Phi$ ), связанные с величинами $\hat{u}_{m}^{n}$, определены следующим образом:

$$
\begin{aligned}
& W^{\mathrm{u}}=\left\{u_{m}^{n} \in \mathcal{L}: \lim _{n \rightarrow-\infty} \mathbf{F}^{n}\left(u_{m}^{n}\right)=\hat{u}_{m}^{n}\right\}, \\
& W^{\mathrm{s}}=\left\{u_{m}^{n} \in \mathcal{L}: \lim _{n \rightarrow+\infty} \mathbf{F}^{n}\left(u_{m}^{n}\right)=\hat{u}_{m}^{n}\right\}
\end{aligned}
$$

и $W^{\mathrm{s}}\left(\hat{u}_{m}^{n}(\varepsilon)\right) \subset W_{\varepsilon}^{\text {сs }}$ и $\mathcal{M}_{\varepsilon}$.

В возмушенной системе получим, что локальные стабильные и нестабильные многообразия $W_{\varepsilon, \text { loc }}^{\mathrm{s}, \mathrm{u}}$ возмушенной точки $\hat{u}_{m}^{n}(\varepsilon)$ оказываются " $\varepsilon$-близки" к соответствуюшим многообразиям для невозмушенной точки $\hat{u}_{m}^{n}=e^{2(-1)^{m}} i \pi$.

Рассмотрим теперь фиксированную седловую точку $\hat{u}_{m}^{n}(\varepsilon)$ на инвариантной плоскости П и будем искать орбиту вне плоскости П, которая гомоклинична к $\hat{u}_{m}^{n}(\varepsilon)$. При этом мы используем геометрические методы для проверки того, что орбиты для почти интегрируемого решеточного уравнения мКд $\Phi$ сохраняют свойство гомоклиничности. Помимо этого метод, с помошю которого ишется ответ на вопрос о том, сушествует ли непустое пересечение множеств $W_{\text {loc }}^{\mathrm{u}}\left(\hat{u}_{m}^{n}(\varepsilon)\right)$ и $W^{\mathrm{s}}\left(\mathcal{M}_{\varepsilon}\right) \subset W_{\varepsilon}^{\mathrm{cs}}$, основывается на измерениях Мельникова [6]. 
Суть метода Мельникова такова. Рассмотрим области $W_{\varepsilon}^{\mathrm{cs}}$ и $W_{\varepsilon, \mathrm{loc}}^{\mathrm{u}}\left(\hat{u}_{m}^{n}(\varepsilon)\right)$ для возмушенного решеточного уравнения мКдФ. Возмушение подбирается таким, чтобы в случае, когда внешние параметры лежат в некотором открытом множестве, сушествовали орбиты, сходящиеся к $\hat{u}_{m}^{n}(\varepsilon)$ при $n \rightarrow \pm \infty$. При этом расстояние между стабильным и нестабильным многообразиями, измеряемое вдоль касательного вектора $\nu_{m}^{n}$, имеет вид [7]

$$
\mathrm{d}=\varepsilon\left(\mathcal{D}^{\mathrm{u}}(0)-\mathcal{D}^{\mathrm{S}}(0)\right)+\mathcal{O}\left(\varepsilon^{2}\right)=\varepsilon \mathrm{M}_{\mathcal{F}}+\mathcal{O}\left(\varepsilon^{2}\right)
$$

где

$$
\mathrm{M}_{\mathcal{F}}:=\sum_{n \in \mathbb{Z}} \sum_{m=0}^{M-1} \nu_{m}^{n}\left(\mathrm{U}_{m}^{n}\right) \mathbf{G}\left(\mathrm{U}_{m}^{n-1}\right)
$$

и величины $\nu_{m}^{n}$ определены формулами (3.19) и должны быть вычислены на гомоклинической орбите $\mathrm{U}_{m}^{n}$.

Функция Мельникова (4.4) при этом имеет вид

$$
\mathrm{M}_{\mathcal{F}}(\mathrm{x}, \tau ; h, M)=\mathrm{bS}_{1}+\mathrm{cS}_{2},
$$

где

$$
\mathrm{S}_{1,2}(\mathrm{x}, \tau ; h, M)=\sum_{n \in \mathbb{Z}} \sum_{m=0}^{M-1} \frac{\delta \mathcal{F}\left(\mathrm{U}_{m}^{n}\right)}{\delta u_{m}^{n}} \mathrm{~g}_{1,2}\left(\mathrm{U}_{m}^{n}\right)=\sum_{n \in \mathbb{Z}} \mathrm{s}_{n}^{(1,2)} .
$$

Полагая $\mathrm{M}_{\mathcal{F}}=0$, получим алгебраическое уравнение, задающее двумерную поверхность в пространстве внешних параметров (b, c):

$$
\mathrm{bS}_{1}+\mathrm{cS}_{2}=0 .
$$

Теперь можно увидеть, что единственными сингулярностями гомоклинических орбит $\mathrm{U}_{m}^{n}$ являются простые полюсы в точках $\tau_{\theta} \in i \pi / 2+i \pi \mathbb{Z}$, а потому функция $\mathrm{U}_{m}^{n}$ аналитична в точках $\tau_{\theta}+h k$ для $k \in \mathbb{Z} \backslash\{0\}$. Вышеприведенная сумма при этом сводится к вычислению вычетов функций $\mathrm{s}^{(i)}, i=1,2$. В частности, функция, которая играет важную роль при вычислении бесконечных сумм в формуле $(4.5),-$ это функция $\chi$ комплексного аргумента, мероморфная в плоскости $\mathbb{C}, T i$-периодичная и ш-периодичная, множество ее полюсов имеет вид $\mathbf{Z} \mathbb{Z}+T i \mathbb{Z}$, при этом все эти полюсы просты и вычеты в них равны единице. Функции s ${ }^{(i)}$ в формуле (4.6):

a) аналитичны на прямой $\mathbb{R}$ и допускают единственную изолированную особую точку в плоскости $\mathbb{C}$;

б) $T i$-периодичны при $T>0$;

в) обладают тем свойством, что

$$
\left|\mathrm{S}^{(i)}(\tau)\right| \leqslant \mathrm{A}_{i} e^{-c_{i}|\operatorname{Re} \tau|}, \quad|\operatorname{Re} \tau| \rightarrow \infty,
$$

при некоторых величинах констант $\mathrm{A}_{i}, c_{i}>0$.

Тогда функция Мельникова $\mathrm{M}_{\mathcal{F}}$ оказьвается аналитичной на прямой $\mathbb{R}$, имеет только изолированные сингулярности на плоскости $\mathbb{C}$ и является дважды периодической с периодами $h, T i$. Более того, функция $\mathrm{M}_{\mathcal{F}}(\tau)$ выражается через сумму следуюшего ряда:

$$
\mathrm{M}_{\mathcal{F}}(\tau ; h)=-\left.\sum_{z \in \mathcal{J}} \mathrm{b} \operatorname{Res} \chi_{T} \mathrm{~s}^{(1)}\right|_{z}+\left.\mathrm{c} \operatorname{Res} \chi_{T} \mathrm{~s}^{(2)}\right|_{z},
$$

где $\mathcal{J}:=\{z \in \mathbb{C}: 0<\operatorname{Im} z<T\}$, а переменная $\tau$ параметризует гомоклинические решения. Теперь можно сформулировать результат. 
ПРЕДЛОЖЕНИЕ 2. Рассмотрим почти интегрируемое решеточное уравнение мКдФ (4.1) с периодическими граничными условиями (1.2). Пусть $M$ - фиксированное четное положительное целое число и $h:=2 \pi / M<1$ - вещественная постоянная. Тогда существует такое число $\varepsilon_{0}>0$, что для любьх фиксированных параметров $\mathrm{w}_{1}, \mathrm{w}_{2}, \mathrm{~b}, \mathrm{c}, \varepsilon$ существует изолированное значение $\tau_{0}$ параметра $\tau$, при котором функиия Мельникова $\mathrm{M}_{\mathcal{F}}$ удовлетворяет условиям

$$
\mathrm{M}_{\mathcal{F}}=0, \quad \partial_{\tau} \mathrm{M}_{\mathcal{F}} \neq 0
$$

При әтом возмущенное решеточное уравнение мКдФ допускает существование гомоклинической орбиты $u_{m}^{n}(\varepsilon)$, которая дважды асимптотически сходится $\kappa$ фиксированной точке $\hat{u}_{m}^{n}(\varepsilon)=e^{2(-1)^{m}} i \pi+\mathcal{O}(\varepsilon)$.

\section{5. ЗАКЛЮЧЕНИЕ}

В данной работе развиты геометрические методы исследования поведения гомоклинических решений решеточного уравнения мКдФ с периодическими граничными условиями при малых диссипативных возмушениях. Важную роль при этом играют преобразования Беклунда, с помошью которых построены гомоклинические орбиты решения решеточного уравнения мКд $\Phi$ в результате элегантной итерации преобразования. Использование критерия Мельникова для дискретных динамических систем позволило показать, что эти орбиты сохраняются при диссипативных возмущениях. При этом функция Мельникова определялась как градиент интеграла движения $\mathcal{F}$, задаваемого с помошью дискретного дискриминанта Флоке, вычисляемого в критических точках.

Благодарности. Автор признателен Институту математических наук Исаака Ньютона (Кембридж) за гостеприимство, оказанное ему во время его пребывания в Кембридже в октябре 2001 г. в рамках программы “Интегрируемые системы". Работа была поддержана EPSRC (грант № GR/R02702/01) и грантом научных сотрудников университета г. Лавборо.

\section{Список литературы}

[1] D. W. McLaughlin, E. A. Overman. Whiskered tori for integrable PDE's: chaotic behavior in near integrable PDE's. In: Surveys in Applied Mathematics. V. 1. Eds. J. B. Keller et al. New York: Plenum, 1995. P. 83-203.

[2] N. Ercolani, M. G. Forest, D. W. McLaughlin. Physica. D. 1990. V. 43. P. 349-384.

[3] V. M. Rothos. Preprint, 2001.

[4] Y. Li, D. W. McLaughlin. Commun. Math. Phys. 1994. V. 162. № 1. P. 175-214.

[5] F. W. Nijhoff, H. W. Capel. Acta Appl. Math. 1995. V. 39. P. 133-158.

[6] В. К. Мельников. Тр. Моск. мат. об-ва. 1965. Т. 12. С. 3-52.

[7] V. M. Rothos. Dyn. Syst. 2001. V. 16. № 3. P. 279-302.

[8] H. W. Capel, F. W. Nijhoff, V. G. Papageorgiou. Phys. Lett. A. 1991. V. 155. P. 377-387.

[9] V. Matveev, M. A. Salle. Darboux Transformations and Solitons. V. 5. Springer Series in Nonlinear Dynamics. New York: Springer, 1991. 